\title{
VP 16-213 in acute myelogenous leukaemia
}

\author{
I. E. SMITH \\ B.Sc., M.R.C.P. \\ H. MACD. ClinK \\ M.D., M.R.C.Path.
}

\author{
M. E. GERKEN \\ M.D. \\ T. J. MCELWAIN \\ M.R.C.P.
}

\section{The Leukaemia Unit, The Institute of Cancer Research and Royal Marsden Hospital, Sutton, Surrey}

\begin{abstract}
Summary
The use of the epipodophyllotoxin VP 16-213 is described in twenty patients with acute myelogenous leukaemia resistant to other chemotherapy. The drug was usually given in 5-day courses of $50 \mathrm{mg} / \mathrm{m}^{2}$ daily and occasionally in 24-hr infusions of $250 \mathrm{mg} / \mathrm{m}^{2}$, on the basis of its phase-activity.

Complete remission was achieved in only two patients: in one of these, remission was maintained with VP 16-213 for 8 months, and in the other for 10 weeks. A partial response was achieved in one other patient. Seventeen patients showed no response. No responses or remissions were achieved when the drug was used in a 24-hr infusion. Side effects were minimal, and the degree of marrow depression much less than for most other agents known to be active in acute myelogenous leukaemia. It was of interest that remission was achieved in one patient without the customary period of marrow hypoplasia. It is suggested that, although VP 16-213 appears to have minimal activity in the dosage used here, improvement might be sought by increasing the dosage, by scheduling the drug in a different way, or by using it in a combination chemotherapy regime.
\end{abstract}

\section{Introduction}

VP 16-213 (Sandoz), a semi-synthetic derivative of the plant product podophyllotoxin, is a cytotoxic drug which a preliminary study has suggested might be active in some human malignancies including acute monocytic and myelomonocytic leukaemia (E.O.R.T.C., 1973).

In a study of VP 16-213 by the European Organisation for Research on Treatment of Cancer, the drug was given daily for a 5-day period. Like V.M. 26, (Stähelin, 1970), it is a phase-active compound, preventing cells from entering mitosis or killing them in pre-mitosis (Dombernowsky and Nissen, 1973). This suggested that greater leukaemic cell kill with

Address for reprints: Dr T. J. McElwain, Royal Marsden Hospital, Sutton, Surrey. relative marrow sparing might be achieved by high dose infusions over a short period, as demonstrated $N$ experimentally for other cytotoxic agents (Bruce, : Meeker and Valeriote, 1966), and later applied clinically with methotrexate (Goldie, Price and Harrap, 1972). This hypothesis is supported by 을 experimental data in which VP 16-213 was more effective against the mouse $L 1210$ leukaemia when $\mathbb{D}$ given in divided doses over a 24-hr period, than in $\frac{0}{2}$ single daily doses (Dombernowsky and Nissen, 1973). This prompted the authors to use VP 16-213 as a high dose 24-hr infusion in some of the $\vec{y}$ patients.

\section{Patients and methods}

VP 16-213 was given to twenty patients with acute myelogenous leukaemia; sixteen of these had acute

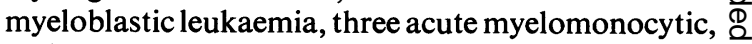
and one acute undifferentiated leukaemia. All were $\overrightarrow{\vec{\rho}}$

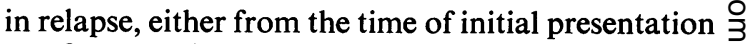
or after a period of remission, and all had failed to $\bar{\partial}$ respond to conventional therapy with various combinations of cytosine arabinoside, daunorubicin, adriamycin and 6-thioguanine (Table 1).

VP 16-213 was given to eighteen of the patients in an i.v. injection of $50 \mathrm{mg} / \mathrm{m}^{2}$ daily for 5 days. This was repeated at two-weekly intervals until it became 8 obvious that the disease was continuing to progress, which in many patients was apparent after only one 옹 course. The drug is presented as a viscous water $\supset$ soluble fluid in $10 \mathrm{ml}$ ampoules and because of the high viscosity of the drug it was necessary to dilute it to $50 \mathrm{ml}$ with normal saline for ease of admini- 5 stration.

In four patients, VP 16-213 was given in a $24-\mathrm{hr}$ N infusion in a dose of $200-250 \mathrm{mg} / \mathrm{m}^{2}$ (i.e. the total ত্ 5 -day dosage) and, in all, seven 24 -hr infusions were 0 given to these four patients (Table 2). Initially, problems arose with drug precipitation when doses ? of $250 \mathrm{mg} / \mathrm{m}^{2}$ (total dose $300-500 \mathrm{mg}$ ) were dissolved 0 in one litre of either normal saline or $5 \%$ dextrose 

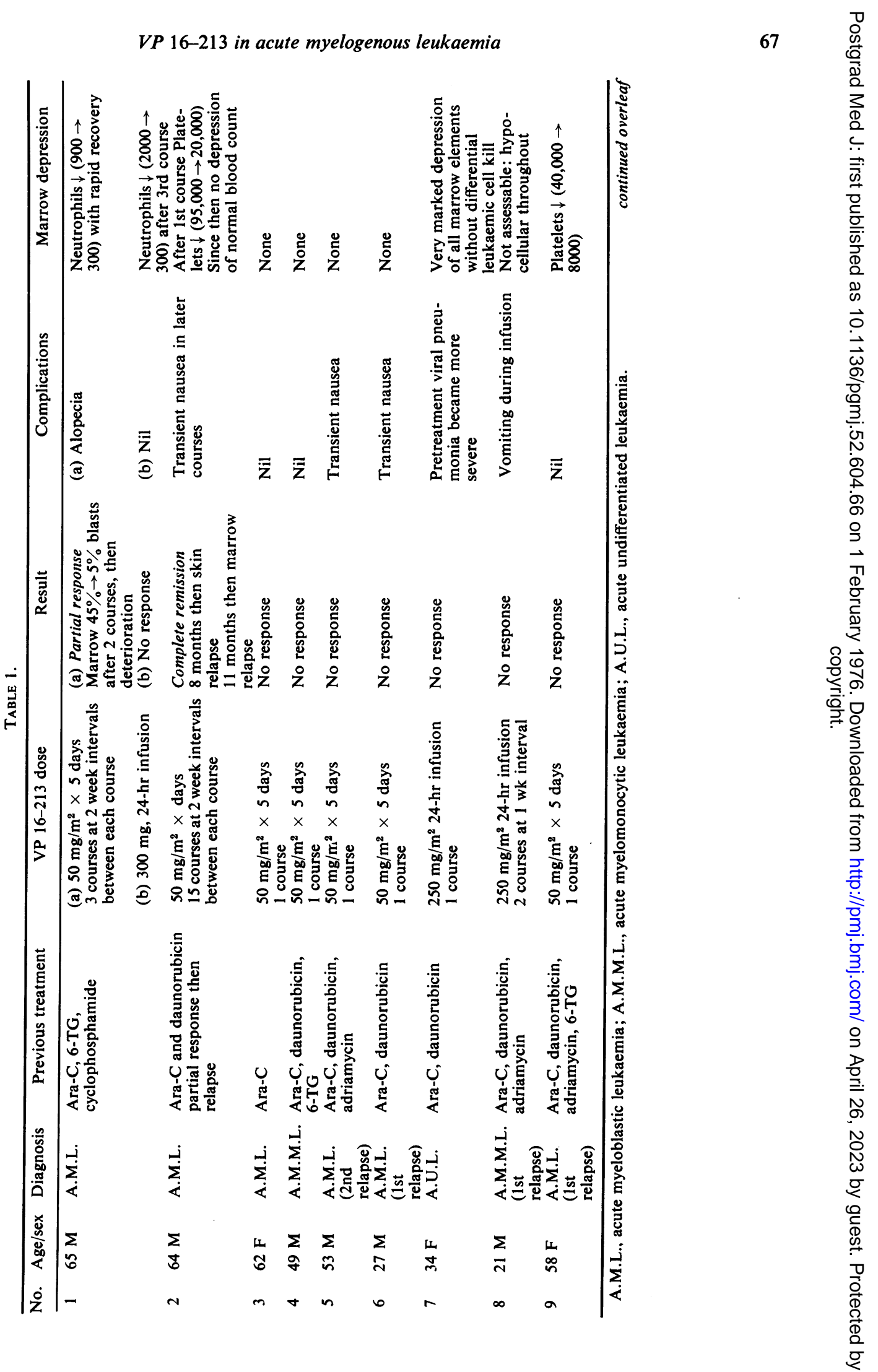


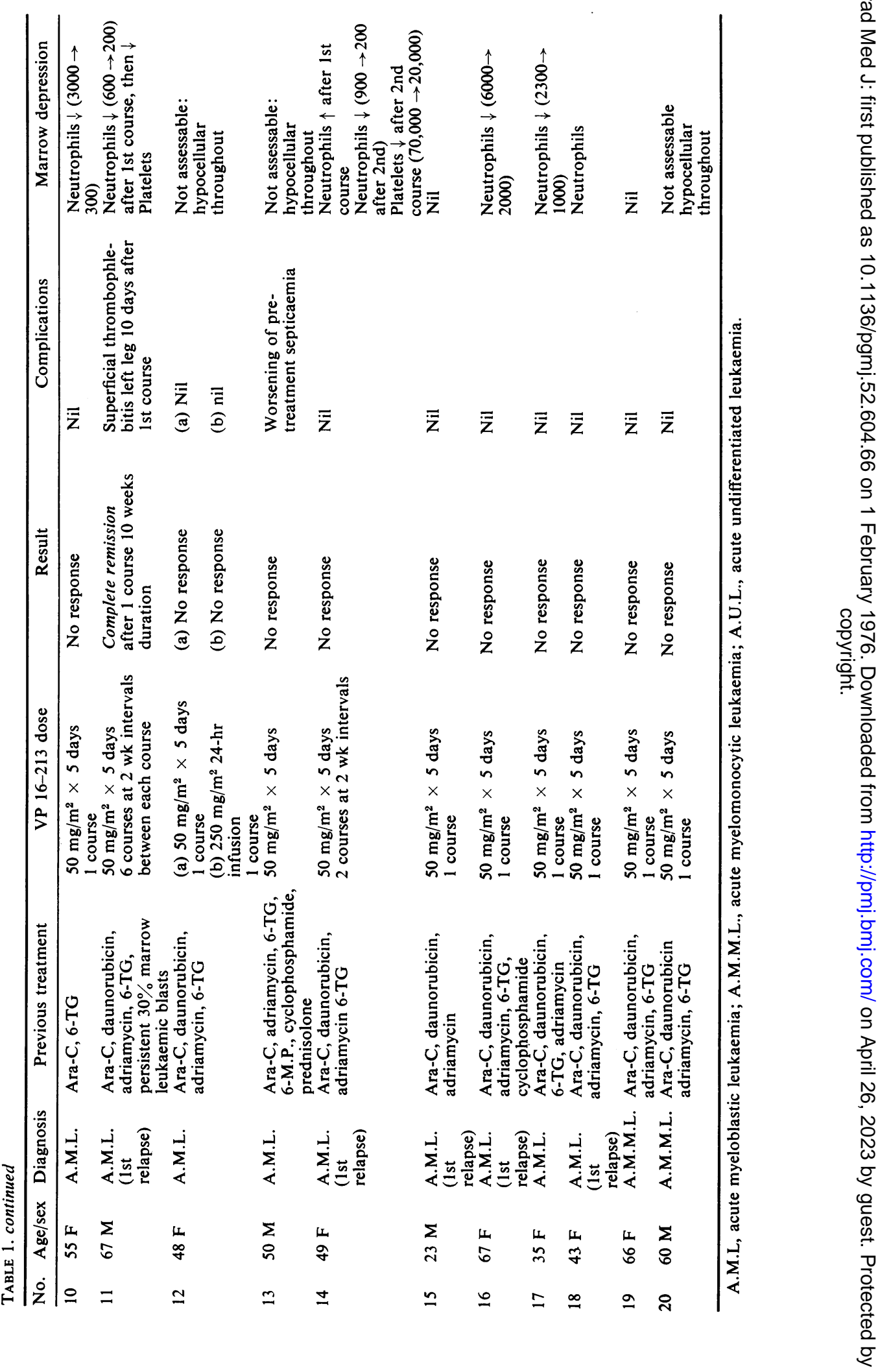


TABLE 2. Side effects and toxicity of VP 16-213

\begin{tabular}{lcc}
\hline & Daily $\times 5$ days & 24-Hour infusion \\
\hline Infection & 1 Septicaemia & 1 Pneumonia/exacerbation \\
Neutrophil depression & $7 / 18$ & $1 / 7$ \\
Platelet depression & $3 / 18$ & $1 / 7$ \\
Nausea & $5 / 18$ & $1 / 7$ \\
Vomiting & - & $1 / 7$ \\
Alopecia & $1 / 18$ & - \\
Thrombophlebitis & $1 / 18$ & - \\
Stomatitis, oral ulceration, & & - \\
gastro-intestinal upset & - & \\
\hline
\end{tabular}

This was overcome by increasing the dilution to 31 of $5 \%$ dextrose, infusing each litre eight-hourly over $24 \mathrm{hr}$.

Peripheral blood counts, plasma urea, electrolytes and protein, serum liver enzymes and bilirubin were monitored before and after treatment. Bone marrow aspirates were done before and after each course of treatment.

Full supportive therapy during treatment, including blood and platelet transfusions and antibiotics where appropriate, was given to all patients.

\section{Results}

Two patients with acute myeloblastic leukaemia achieved complete haematological and clinical remission after treatment with VP 16-213. The first of these (Case 2, Table 1), achieved remission after one 5-day course of therapy, without depression of peripheral blood count. He was maintained in remission on 5-day courses at two-weekly intervals for 9 months before developing leukaemic skin infiltrates. He subsequently developed marrow relapse 11 months after the start of VP 16-213 therapy. His presenting marrow aspirate showed basophilic plasmocytoid blast cells with a low nuclear/cytoplasmic ratio and unusual appearance, but three haematologists independently confirmed a morphological diagnosis of acute myeloblastic leukaemia. No side effects were experienced from courses of maintenance treatment given at 2-weekly intervals for 9 months, except for occasional transient nausea on the first and second day of each course.

The second patient (Case 12, Table 1), also achieved remission after one 5-day course of therapy, with depression of blood neutrophils but not of platelets. He was maintained in remission on 5-day courses at 2-weekly intervals for 10 weeks before haematological relapse.

One patient (Case 1, Table 1) with acute myeloblastic leukaemia showed a partial response to VP 16-213. His marrow aspirate showed a fall in leukaemic blasts from 50 to $7 \%$ with rising peripheral platelet count after two courses of treatment, but the effect was short-lived and the marrow once more deteriorated after the third course of treatment.
None of the remaining seventeen patients showed any response to VP 16-213 therapy and in particular no response was seen to any of the seven 24-hr infusions.

In general, treatment was well tolerated with a remarkably low incidence of toxicity (Table 2). Bone marrow depression due to VP 16-213 was rarely severe, although many patients had severely hypoplastic bone marrows before therapy, because of disease and previous treatment. A fall in neutrophil count was seen in eight patients and a fall in platelet count in four; on the other hand, the neutrophil count rose in four instances after therapy, and the platelets rose in two. It was often hard to interpret whether these results were directly attributable to VP 16-213 or to prolonged effects of previous therapy.

Nausea occurred transiently in five patients but was associated with vomiting in only one, and this was during a 24-hr infusion. Other gastro-intestinal disturbances, including oral ulceration, were not seen. Reversible alopecia occurred in one man. No disturbance in plasma electrolytes, urea, serum liver enzymes or bilirubin attributable to VP 16-213 was seen.

\section{Discussion}

This study suggests that VP 16-213 is only rarely effective in the treatment of acute myelogenous leukaemia, as a single agent given either as a course of five daily injections or in a single high dose infusion over $24 \mathrm{hr}$. It was also not possible to confirm in the three patients with acute myelomonocytic leukaemia the E.O.R.T.C. (1973) finding that the drug is particularly effective in this type of leukaemia. Nevertheless, the complete remission obtained in one patient (Case 2, Table 1) was striking in that it was achieved without a significant degree of marrow suppression at any stage. This is very unusual with other current chemotherapy effective against acute myelogenous leukaemia. It was of interest that this patient had a morphologically unusual myeloblastic leukaemia.

In the dosage described, VP 16-213 rarely produced severe marrow toxicity or unpleasant side 
effects, in contrast to most other anti-leukaemic agents. This, despite its low remission rate, perhaps justifies the continuing use of the drug as a last resort, in that at least it is unlikely to worsen the patient's condition.

There are possible ways in which the effectiveness of VP 16-213 might be improved. Firstly, the relatively moderate bone marrow toxicity suggests that the dose of the drug might be increased without life-threatening consequences, particularly if it were given as first line treatment, without a marrow made hypoplastic by previous chemotherapy. Secondly, its phase-activity and increased efficiency with scheduling in the $\mathrm{L} 1210$ experimental leukaemia (Dombernowsky and Nissen, 1973) suggests that appropriate scheduling might produce better clinical effect, despite the lack of response to a 24-hr infusion in the authors' experience. Finally, VP 16-213 might be more effective used in combination or sequential chemotherapy.

\section{Acknowledgments}

We should like to thank Sandoz Products, and in particular Dr Graham Kennedy, for providing VP 16-213 and for help and encouragement in the study. We thank Professor F. G. J.
Hayhoe and Dr C. Holman for reviewing the marrow slides of one patient (Case 2). We express our gratitude to Sister J. Lynch and nursing staff in the Leukaemia Unit at the Royal Marsden Hospital, Sutton, for their care and attention to the patients in this study.

\section{References}

Bruce, W.R., Meeker, B.E. \& Valeriote, F.A. (1966) Comparison of the sensitivity of normal haematopoietic and transplanted lymphoma colony forming cells to chemotherapeutic agents administered in vivo. Journal of the National Cancer Institute, 37, 233.

Dombernowsky, P. \& NisSen, N.I. (1973) Schedule dependency of the antileukaemic activity of the podophyllotoxin derivative VP 16-213 (NSC-141540) in L 1210 leukaemia. Acta pathologica microbiologica scandinavica. Section A, 81,715 .

EUROPEAN ORgaNisation FOR RESEARCH ON THE TREATMENT of Cancer, Clinical Screening Group (1973) Epipodophyllotoxin VP 16-213 in treatment of acute leukaemias, haematosarcomas, and solid tumours. British Medical Journal, 3, 199.

Goldie, J.H., Price, L.A. \& Harrap, K.R. (1972) Methotrexate toxicity: correlation with duration of administration, plasma levels, dose and excretion pattern. European Journal of Cancer, 8, 409.

STÄHELIN, H. (1970) 4'-demethyl-epipodophyllotoxin thenylidene glucoside (V.M. 26), a podophyllum compound with a new mechanism of action. European Journal of Cancer, 6 , 303. 